\title{
Age comparison of treatment adherence with antipsychotic medications among individuals with bipolar disorder
}

\author{
Martha Sajatovic $^{1 *}$, Frederic C. Blow ${ }^{2,3}$, Helen C. Kales ${ }^{2,4}$, Marcia Valenstein ${ }^{2,3}$, \\ Dara Ganoczy ${ }^{2}$ and Rosalinda V. Ignacio ${ }^{2,3}$ \\ ${ }^{1}$ Psychiatry and Epidemiology and Biostatistics, Case Western Reserve University School of Medicine, \\ Cleveland, OH, USA \\ ${ }^{2}$ Serious Mental Illness Treatment Research and Evaluation Center (SMITREC), Health Services Research \\ and Development, Ann Arbor VA Healthcare System, MI, USA \\ ${ }^{3}$ Department of Psychiatry, University of Michigan, Ann Arbor, MI, USA \\ ${ }^{4}$ Section of Geriatric Psychiatry, University of Michigan, Ann Arbor, MI, USA
}

\section{SUMMARY}

Background Few studies have evaluated medication adherence among older $v s$ younger individuals with bipolar disorder (BPD). We compared adherence with antipsychotic medication among older (age 60 and older) and younger individuals using a large case registry $(n=73,964)$.

Methods Adherence was evaluated using the medication possession ratio (MPR) for patients receiving antipsychotic medication.

Results Twenty six thousand five hundred and thirty younger individuals (mean age 46.9) and 6,461 older individuals (mean age 69.2) were prescribed antipsychotic medication. Among older individuals, $61.0 \%(n=3,350)$ were fully adherent, while $19.0 \%(n=1,043)$ were partially adherent and $20.0 \%(n=1,098)$ were non-adherent. Among younger individuals, $49.5 \%(n=10,644)$ were fully adherent, while $21.8 \%(n=4,680)$ were partially adherent, and $28.7 \%(n=6,170)$ were non-adherent. As with younger patients, comorbid substance abuse and homelessness predicted non-adherence among older patients with BPD.

Conclusion Older individuals with BPD were more adherent with antipsychotic medications compared to younger individuals. However, a substantial proportion (approximately 39\%) of older patients with BPD still have difficulties with adherence. Copyright (C) 2007 John Wiley \& Sons, Ltd.

KEY WORDS - treatment adherence; antipsychotic medications; bipolar disorder; elderly; geriatric

\section{INTRODUCTION:}

An emerging literature has identified treatment non-adherence as a pervasive problem that leads to illness relapse, hospitalization and other negative sequelae (Perlick et al., 2004). Studies evaluating medication non-adherence among patients with BPD found median rates of $41 \%$ (Lingam and Scott, 2002)

*Correspondence to: Dr M. Sajatovic, Department of Psychiatry, University Hospitals of Cleveland, 11100 Euclid Avenue, Cleveland, OH 44106, USA. E-mail: martha.sajatovic@uhhs.com and $42 \%$ (Perlick et al., 2004). Medication adherence is a complex phenomenon that appears to be influenced by a number of patient, provider, and environmental factors (Aagaard et al., 1988; Aagaard and Vestergaard, 1990; Colom and Vieta, 2002; Berk et al., 2004). Patient characteristics that appear to be associated with adherence in BPD include age, marital status, gender, and educational level (Aagard et al., 1988; Lingam and Scott, 2002; Berk et al., 2004). Concurrent psychiatric disorders also appear to influence adherence (Aagaard and Vestergaard, 1990; Keck et al., 1997; Perlick et al., 
2004). However, few studies have evaluated older vs younger individuals with BPD with respect to treatment adherence. Additionally, there is limited information on adherence with antipsychotic medications, a class of drugs that is being used with increasing frequency to treat bipolar illness. Using a large case registry $(n=73,964)$, we examined adherence with antipsychotic medication among veterans with BPD within a one-year period [Federal Fiscal Year 2003 (FY03), 1 October 2002-30 September 2003]. The aim of the study was to compare treatment adherence among older individuals (age 60 and older) with younger individuals.

\section{METHODS}

\section{Subjects}

Registry patients were identified using the VA's National Psychosis Registry (NPR), which regularly incorporates data from the VA Patient Treatment File (PTF), census data files, and Outpatient Care Files (OPC) and the VA Pharmacy Benefits Management Group. We identified patients with bipolar diagnosis in the NPR using ICD9-CM codes 296.0, 296.1, 296.4, 296.5, 296.6, 296.7, and 296.8. Individuals were included if they had at least one qualifying diagnosis during Federal Fscal Year 2003 (FY03). In the event that patients received more than one diagnosis over time, individuals were assigned to the diagnosis which appeared in the greatest number of episodes of care. Ties were resolved using a rank ordering of: (1) schizophrenia; (2) bipolar disorder; and (3) other psychosis. A full report of the Psychosis Registry may be viewed at: http://www.va.gov/annarbor-hsrd.

\section{Measurement of adherence}

Medication adherence was evaluated using the medication possession ratio (MPR) for patients receiving any antipsychotic medication. The MPR is the ratio of the "number of days supply of medication that a patient has received' to the 'number of days supply that they should have received' had they been taking medication as prescribed. An MPR of 1 or $100 \%$ indicates that the patient has received all the medication needed to take their antipsychotic medication as prescribed, whereas an MPR of $0.5 \%$ or $50 \%$ indicates that the patient has received medication sufficient to take only half of the prescribed dose.

The MPR was calculated among patients with at least 90 outpatient days of observation during the fiscal year. The MPR was calculated for the time period of FY03 following the date of the patient's first antipsychotic medication fill of the year. Days spent in institutional settings were subtracted from the numbers of days supply the patient 'should have received' in order to take their medication as prescribed. MPR calculations were limited to individuals who were taking no more than two antipsychotic medications during the fiscal year. In cases where an individual was on two antipsychotic medications, a weighted average of the two MPRs was calculated. The MPR, a measure of prescription refills, has been widely utilized in both medical and psychiatric settings as a proxy measure of treatment adherence (Al-Zakwani et al., 2003; Hertz et al., 2005; Patel et al., 2005; Sanchez et al., 2005; Yu et al., 2005), including assessment of adherence in populations with serious mental illness, and in bipolar populations specifically (Valenstein et al., 2002; Gianfrancesco et al., 2006).

Antipsychotics prescribed in the VA during FY03 were conventional antipsychotic agents as well as the atypical agents clozapine, risperidone, olanzapine, quetiapine, ziprasidone and aripiprazole. Long-acting injectable medications and medications prescribed for use in inpatient settings were not included in analysis.

\section{Adherence intensity}

Patients' degree of adherence was evaluated in three clinically relevant categories. Individuals who were fully adherent with antipsychotic medication had MPRs greater than 0.8 . Individuals who were partially adherent with antipsychotic medication had MPRs of more than 0.5 and less than or equal to 0.8 . Individuals who were considered to be non-adherent with medication had MPRs of less than or equal to 0.5. Unlike some other previous reports (Al-Zakwani et al., 2003), a maximum ratio of 1 was not applied, as this would bias comparisons against agents with a relatively high adherence intensity. While MPRs greater than 1 may also reflect over-prescribers (Gilmer et al., 2004) this cannot be determined solely from claims data. For example, MPRs greater than 1 were possible in cases where prescriptions may not have been completely used due to interim changes in dosage.

\section{Statistical analysis}

Descriptive statistics were used to characterize demographic and clinical characteristics of all patients with BPD. A multiple logistic model was used to compare characteristics of older vs younger patients 
with BPD. Multinomial multiple logistic regression models compared characteristics of patients with full or partial adherence to patients who were nonadherent to antipsychotic medication, controlling for the effects of gender, age, race/ethnicity, marital status, previous psychiatric hospitalization, posttraumatic stress disorder (PTSD), homelessness, and concurrent substance abuse. PTSD and substance abuse were selected as covariates of particular relevance given the overwhelmingly male sample and the fact that relatively high rates of combat-related PTSD are seen in veteran populations. Characteristics associated with adherence were examined separately for the younger and older age groups. In addition, a model including both age groups was analyzed to examine interactions between age group and demographic and clinical variables, adjusting the significance tests for multiple comparisons. Wilcoxon tests were used to compare mean MPRs for patients receiving one $v s$ two antipsychotics, for patients receiving atypical vs conventional antipsychotics, for patients receiving clozapine $v s$ other atypical antipsychotics, and for older $v s$ younger patients.

\section{RESULTS}

There were 56,572 (76.5\% of the total bipolar group) individuals under age 60 and 17,388 (23.5\%) individuals age 60 or older. Compared to younger individuals, older individuals were more likely to be Caucasian (Wald $\chi^{2}=128.6, \mathrm{df}=1, p<0.0001$ ) and married (Wald $\chi^{2}=149.7, \mathrm{df}=1, p<0.0001$ ). Younger individuals were more likely to have comorbid substance abuse (Wald $\left.\chi^{2}=511.5, \mathrm{df}=1, p<0.0001\right)$, comorbid PTSD (Wald $\chi^{2}=420.2, \mathrm{df}=1, p<0.0001$ ) and be homeless (Wald $\chi^{2}=95.6, \mathrm{df}=1, p<0.0001$ ) compared to older individuals (Table 1). There were 26,530 (46.9\%) individuals prescribed antipsychotic medications among the younger group (mean age 46.9, $\mathrm{SD} \pm 8.1$ years) compared to $6,461(37.2 \%)$ among the

Table 1. Characteristics of veterans with bipolar disorder in FY03, stratified by age group

\begin{tabular}{|c|c|c|c|c|}
\hline Characteristic & $\begin{array}{c}\text { All veterans with } \\
\text { bipolar disorder } \\
n(\%)\end{array}$ & $\begin{array}{c}\text { All veterans with } \\
\text { bipolar disorder } \\
\text { Age }<60 \text { years } \\
n(\%)\end{array}$ & $\begin{array}{c}\text { All veterans with } \\
\text { bipolar disorder } \\
\text { Age } \geq 60 \text { years } \\
n(\%)\end{array}$ & $\begin{array}{l}\text { Test of significance between groups } \\
\text { (controlling for other covariates) }\end{array}$ \\
\hline Number of patients & 73,964 & $56,572(76.5)$ & $17,388(23.5)$ & \\
\hline Mean age (SD) & $52.3(12.5)$ & $47.0(8.3)$ & $69.5(7.1)$ & Wilcoxon Test $Z=199.8, p<0.0001^{*}$ \\
\hline \multicolumn{5}{|r|}{ (1) } \\
\hline Male & $65,212(88.2)$ & $48,559(85.8)$ & $16,650(95.8)$ & \multirow[t]{2}{*}{ Wald $\chi^{2}=300.3, p<0.0001, \mathrm{df}=11$} \\
\hline Female & $8,752(11.8)$ & $8,013(14.2)$ & $738(4.2)$ & \\
\hline \multicolumn{5}{|l|}{ Ethnicity } \\
\hline White & $46,251(62.5)$ & $34,395(60.8)$ & $11,856(68.2)$ & \multirow[t]{6}{*}{ Wald $\chi^{2}=128.6, p<0.0001, \mathrm{df}=1$} \\
\hline Black & $7,694(10.4)$ & $6,904(12.2)$ & $790(4.5)$ & \\
\hline Hispanic & $2,249(3.0)$ & $1,814(3.2)$ & $435(2.5)$ & \\
\hline American Indian & $239(0.3)$ & $216(0.4)$ & $23(0.1)$ & \\
\hline Asian & $172(0.2)$ & $142(0.3)$ & $30(0.2)$ & \\
\hline Unknown & $17,359(23.5)$ & $13,101(23.2)$ & $4,254(24.5)$ & \\
\hline \multicolumn{5}{|l|}{ Marital status } \\
\hline Never married & $15,386(21.0)$ & $13,637(24.3)$ & $1,748(10.1)$ & \multirow[t]{4}{*}{ Wald $\chi^{2}=149.7, p<0.0001, \mathrm{df}=1$} \\
\hline Married & $27,651(37.7)$ & $18,813(33.6)$ & $8,838(51.2)$ & \\
\hline Divorced/separated & $27,751(37.9)$ & $22,619(40.4)$ & $5,131(29.7)$ & \\
\hline Widowed & $2,497(3.4)$ & $949(1.7)$ & $1,548(9.0)$ & \\
\hline \multicolumn{5}{|l|}{ Substance use disorder } \\
\hline Yes & $23,484(31.8)$ & $21,152(37.4)$ & 2,331 (13.4) & \multirow[t]{2}{*}{ Wald $\chi^{2}=511.5, p<0.0001, \mathrm{df}=11$} \\
\hline No & $50,480(68.3)$ & $35,420(62.6)$ & $15,057(86.6)$ & \\
\hline \multicolumn{5}{|l|}{ PTSD diagnosis } \\
\hline Yes & $15,721(21.3)$ & $13,921(24.6)$ & $1,800(10.4)$ & \multirow[t]{2}{*}{ Wald $\chi^{2}=420.2, p<0.0001, \mathrm{df}=1$} \\
\hline No & $58,243(78.8)$ & $42,651(75.4)$ & $15,588(89.7)$ & \\
\hline \multicolumn{5}{|l|}{ Homelessness } \\
\hline Yes & $10,176(13.8)$ & $9,447(16.7)$ & $729(4.2)$ & \multirow[t]{2}{*}{ Wald $\chi^{2}=95.6, p<0.0001, \mathrm{df}=1$} \\
\hline No & $63,788(86.2)$ & $47,125(83.3)$ & $16,659(95.8)$ & \\
\hline \multicolumn{5}{|c|}{$\begin{array}{l}\text { Psychiatric hospitalization } \\
\text { in FY02 }\end{array}$} \\
\hline Yes & $10,236(13.8)$ & $8,689(15.4)$ & $1,547(8.9)$ & \multirow[t]{2}{*}{ Wald $\chi^{2}=0.6, p=0.4333, \mathrm{df}=1$} \\
\hline No & $63,724(86.2)$ & $47,883(84.6)$ & $15,841(91.1)$ & \\
\hline
\end{tabular}

*Comparing mean age between groups: 47.0 vs 69.5 . 
older group (mean age $69.2 \pm 7.0$ years) $\left(\chi^{2}=510.4\right.$, $\mathrm{df}=1, p<0.0001)$.

Among younger adults prescribed antipsychotics, $49.5 \%(n=10,644)$ were fully adherent, while $21.8 \%$ $(n=4,680)$ were partially adherent, and $28.7 \%$ $(n=6,170)$ were non-adherent (Table 2). Among older adults prescribed antipsychotics, $61.0 \%(n=$ $3,350)$ were fully adherent, while $19.0 \%(n=1,043)$ were partially adherent and $20.0 \%(n=1,098)$ were non-adherent (Table 3). Non-adherent older adults were more likely to have substance abuse [Odds Ratio $(\mathrm{OR})=1.38$, 95\% Confidence Intervals (CI):1.13, $1.68]$ and be homeless ( $\mathrm{OR}=1.44,95 \% \mathrm{CI}: 1.05,1.97)$ compared to older adults who were fully or partially adherent, while non-adherent younger adults were more likely to be of minority ethnicity $(\mathrm{OR}=1.49$, $95 \%$ CI:1.38, 1.61), have comorbid substance abuse $(\mathrm{OR}=1.3095 \% \mathrm{CI}: 1.20,1.40)$ and be homeless $(\mathrm{OR}=1.53,95 \% \mathrm{CI}: 1.39,1.67)$ compared to younger adults who were fully or partially adherent. The interaction effects on adherence between age group and gender, race/ethnicity, marital status, previous psychiatric hospitalization, homelessness, comorbid PTSD, and substance abuse were also examined. With the exception of race/ethnicity, these interactions were non-significant, suggesting that demographic and clinical factors have similar associations with adherence across age groups. However, the race/ethnicity and age-group interaction was significant (Wald $\left.\chi^{2}=22.0, \mathrm{df}=2, p<0.0001\right)$. This indicates that being in the older age group was associated with a greater increase in antipsychotic adherence among African Americans than among white patients $(\mathrm{OR}=1.58,95 \% \mathrm{CI}: 1.17,2.14)$.

Table 4 demonstrates adherence intensity for individuals with BPD. Younger individuals had a mean MPR on any antipsychotic of $0.74( \pm 0.37)$, while older individuals had a mean MPR of 0.82 ( \pm 0.34 ) (Wilcoxon Test, $Z=14.4, p<0.0001$ ). The majority of individuals prescribed antipsychotics in

Table 2. Intensity of adherence among individuals with bipolar disorder, age $<60$ years

\begin{tabular}{|c|c|c|c|c|}
\hline & $\begin{array}{c}\text { Fully adherent }^{\mathrm{a}} \\
n(\%)\end{array}$ & $\begin{array}{c}\text { Partially adherent } \\
n(\%)\end{array}$ & $\begin{array}{c}\text { Non-adherent }{ }^{\mathrm{c}} \\
n(\%)\end{array}$ & $\begin{array}{l}\text { Test of significance between groups } \\
\text { (controlling for other covariates) }\end{array}$ \\
\hline Number of patients & $10,644(49.5)$ & $4,680(21.8)$ & $6,170(28.7)$ & \\
\hline Mean age (SD) & $48.0(7.6)$ & $46.9(8.0)$ & $45.6(8.6)$ & Wald $\chi^{2}=150.4, p<0.0001, \mathrm{df}=1$ \\
\hline \multicolumn{5}{|l|}{ Gender } \\
\hline Male & $9,137(85.8)$ & $4,011(85.7)$ & $5,287(85.7)$ & \multirow{2}{*}{ Wald $\chi^{2}=3.2, p=0.0726, \mathrm{df}=1$} \\
\hline Female & $1,507(14.2)$ & $669(14.3)$ & $883(14.3)$ & \\
\hline \multicolumn{5}{|l|}{ Ethnicity } \\
\hline White & 7,509 (70.6) & $3,027(64.7)$ & $3,621(58.7)$ & \multirow[t]{6}{*}{ Wald $\chi^{2}=97.3, p<0.0001, \mathrm{df}=1$} \\
\hline Black & $1,000(9.4)$ & $706(15.1)$ & $1,051(17.0)$ & \\
\hline Hispanic & $372(3.5)$ & $168(3.6)$ & $237(3.8)$ & \\
\hline American Indian & $49(0.5)$ & $16(0.3)$ & $21(0.3)$ & \\
\hline Asian & $26(0.2)$ & $8(0.2)$ & $15(0.2)$ & \\
\hline Unknown & $1,688(15.9)$ & $755(16.1)$ & $1,225(19.9)$ & \\
\hline \multicolumn{5}{|l|}{ Marital status } \\
\hline Never married & 2,555 (24.2) & $1,118(24.0)$ & $1,604(26.2)$ & \multirow[t]{4}{*}{ Wald $\chi^{2}=0.5, p=0.4971, \mathrm{df}=1$} \\
\hline Married & $3,735(35.3)$ & $1,538(33.0)$ & $1,872(30.6)$ & \\
\hline Divorced/separated & $4,111(38.9)$ & $1,921(41.3)$ & $2,546(41.6)$ & \\
\hline Widowed & $168(1.6)$ & $80(1.7)$ & $95(1.6)$ & \\
\hline \multicolumn{5}{|l|}{ Substance use disorder } \\
\hline Yes & 3,554 (33.4) & $1,921(41.1)$ & 2,742 (44.4) & \multirow[t]{2}{*}{ Wald $\chi^{2}=46.7, p<0.0001, \mathrm{df}=1$} \\
\hline No & $7,090(66.6)$ & $2,759(59.0)$ & $3,428(55.6)$ & \\
\hline \multicolumn{5}{|l|}{ PTSD diagnosis } \\
\hline Yes & $2,905(27.3)$ & $1,390(29.7)$ & $1,776(28.8)$ & \multirow[t]{2}{*}{ Wald $\chi^{2}=3.5, p=0.0629, \mathrm{df}=1$} \\
\hline No & $7,739(72.7)$ & $3,290(70.3)$ & $4,394(71.2)$ & \\
\hline \multicolumn{5}{|l|}{ Homelessness } \\
\hline Yes & 1,213 (11.4) & $782(16.7)$ & $1,286(20.8)$ & \multirow[t]{2}{*}{ Wald $\chi^{2}=81.1, p<0.0001, \mathrm{df}=1$} \\
\hline No & $9,431(88.6)$ & $3,898(83.3)$ & $4,884(79.2)$ & \\
\hline \multicolumn{5}{|c|}{ Psychiatric hospitalization in FY02 } \\
\hline Yes & 2,071 (19.5) & 1,027 (21.9) & $1,354(21.9)$ & \multirow[t]{2}{*}{ Wald $\chi^{2}=0.3, p=0.5674, \mathrm{df}=1$} \\
\hline No & $8,573(80.5)$ & $3,653(78.1)$ & $4,816(78.1)$ & \\
\hline
\end{tabular}

${ }^{a}$ Adherent with $>80 \%$ of medication.

${ }^{\mathrm{b}}$ Adherent with $>50-80 \%$ of medication.

${ }^{\mathrm{c}}$ Adherent with less than or equal to $50 \%$ of medication. 
Table 3. Intensity of adherence among individuals with bipolar disorder, age $\geq 60$ years

\begin{tabular}{|c|c|c|c|c|}
\hline & $\begin{array}{c}\text { Fully adherent }{ }^{\mathrm{a}} \\
n(\%)\end{array}$ & $\begin{array}{c}\text { Partially adherent }{ }^{\mathrm{b}} \\
n(\%)\end{array}$ & $\begin{array}{c}\text { Non-adherent }{ }^{\mathrm{c}} \\
n(\%)\end{array}$ & $\begin{array}{l}\text { Test of significance between groups } \\
\text { (controlling for other covariates) }\end{array}$ \\
\hline Number of patients & $3,350(61.0)$ & $1,043(19.0)$ & $1,098(20.0)$ & \\
\hline Mean age (SD) & $69.2(6.9)$ & $69.2(7.0)$ & $69.0(7.3)$ & Wald $\chi^{2}=0.1, p=0.8108, \mathrm{df}=1$ \\
\hline \multicolumn{5}{|l|}{ Gender } \\
\hline Male & $3,181(95.0)$ & $993(95.2)$ & $1,042(94.9)$ & \multirow[t]{2}{*}{ Wald $\chi^{2}=0.1, p=0.7089, \mathrm{df}=1$} \\
\hline Female & $169(5.0)$ & $50(4.8)$ & $56(5.1)$ & \\
\hline \multicolumn{5}{|l|}{ Ethnicity } \\
\hline White & $2,473(73.8)$ & $718(68.8)$ & $800(72.9)$ & \multirow[t]{6}{*}{ Wald $\chi^{2}=0.4, p=0.5029, \mathrm{df}=1$} \\
\hline Black & $168(5.0)$ & $65(6.2)$ & $64(5.8)$ & \\
\hline Hispanic & $110(3.3)$ & $47(4.5)$ & $29(2.6)$ & \\
\hline American Indian & $5(0.2)$ & $2(0.2)$ & 0 & \\
\hline Asian & $4(0.1)$ & $2(0.2)$ & $3(0.3)$ & \\
\hline Unknown & $590(17.6)$ & $209(20.0)$ & $202(18.4)$ & \\
\hline \multicolumn{5}{|l|}{ Marital status } \\
\hline Never married & $368(11.0)$ & $92(8.9)$ & $106(9.7)$ & \multirow[t]{4}{*}{ Wald $\chi^{2}=0.6, p=0.4581, \mathrm{df}=1$} \\
\hline Married & $1,673(50.2)$ & $508(49.2)$ & $512(46.9)$ & \\
\hline Divorced/separated & 973 (29.2) & $332(32.2)$ & $384(35.2)$ & \\
\hline Widowed & $318(9.5)$ & $100(9.7)$ & $89(8.2)$ & \\
\hline \multicolumn{5}{|l|}{ Substance use disorder } \\
\hline Yes & 459 (13.7) & $154(14.8)$ & $215(19.6)$ & \multirow[t]{2}{*}{ Wald $\chi^{2}=10.1, p=0.0015, \mathrm{df}=1$} \\
\hline No & $2,891(86.3)$ & $889(85.2)$ & $883(80.4)$ & \\
\hline \multicolumn{5}{|l|}{ PTSD diagnosis } \\
\hline Yes & 404 (12.1) & $113(10.8)$ & $139(12.7)$ & \multirow[t]{2}{*}{ Wald $\chi^{2}=0.3, p=0.5914, \mathrm{df}=1$} \\
\hline No & 2,946 (87.9) & $930(89.2)$ & $959(87.3)$ & \\
\hline \multicolumn{5}{|l|}{ Homelessness } \\
\hline Yes & $114(3.4)$ & $56(5.4)$ & $75(6.8)$ & \multirow[t]{2}{*}{ Wald $\chi^{2}=5.2, p=0.0225, \mathrm{df}=1$} \\
\hline No & $3,236(96.6)$ & 987 (94.6) & $1,023(93.2)$ & \\
\hline \multicolumn{5}{|c|}{ Psychiatric hospitalization in FY02 } \\
\hline Yes & 493 (14.7) & $151(14.5)$ & $180(16.4)$ & \multirow[t]{2}{*}{ Wald $\chi^{2}=0.3, p=0.5693, \mathrm{df}=1$} \\
\hline No & $2,857(85.3)$ & $892(85.5)$ & 918 (83.6) & \\
\hline
\end{tabular}

${ }^{\mathrm{a}}$ Adherent with $>80 \%$ of medication.

${ }^{\mathrm{b}}$ Adherent with $>50-80 \%$ of medication.

${ }^{\mathrm{c}}$ Adherent with less than or equal to $50 \%$ of medication.

both older $(91.9 \%)$ and younger $(95.4 \%)$ groups received atypical antipsychotics. MPRs were higher for patients on two antipsychotics compared to those taking single antipsychotics for both younger individuals $(Z=6.1, p<0.0001)$ and older individuals $(Z=2.6, p=0.0082)$. For individuals on only one antipsychotic, MPRs were higher for conventional antipsychotics compared to atypical antipsychotics for both younger $(Z=5.7, p<0.0001)$ and older $(Z=2.6$, $p=0.0094)$ individuals.

\section{DISCUSSION}

In this large sample, antipsychotic medications were prescribed less often to treat older individuals with BPD (37.2\%), compared to younger individuals with BPD $(46.9 \%)$. In common with earlier reports on treatment adherence with traditional mood stabilizers, older adults with BPD were more adherent with antipsychotic medications compared to younger adults with BPD (Perlick et al., 2004). Preliminary work by this group of investigators (Valenstein and Sajatovic, in press) suggests that non-adherence rates among bipolar populations appear rather similar across compounds including lithium, anticonvulsants and antipsychotic drugs. However, approximately $39 \%$ of older adults had adherence difficulties, indicating that this remains a pressing clinical issue for geriatric populations. As with younger adults, substance abuse and being homeless predicted non-adherence among older adults with BPD. It has been reported that younger and older bipolar populations have cognitive impairments (Nehra et al., 2006; Robinson et al., 2006; Young et al., 2006) that potentially could impact treatment adherence. Older adults may be more likely than younger individuals to have other individuals such as family re-filling their medications, thus contributing at least in part, to their slightly greater adherence rates.

The majority of bipolar patients prescribed antipsychotic medications received atypical agents. Clearly, atypical antipsychotic medications have 
Table 4. Adherence with antipsychotic medication among individuals with bipolar disorder

\begin{tabular}{|c|c|c|c|c|c|}
\hline & \multicolumn{2}{|l|}{$<60$ years } & \multicolumn{2}{|l|}{$\geq 60$ years } & \multirow{2}{*}{$\begin{array}{l}\text { Wilcoxon Test of } \\
\text { significance between } \\
\text { age groups }\end{array}$} \\
\hline & $\begin{array}{c}\text { Number of individuals } \\
n(\%)\end{array}$ & $\begin{array}{c}\text { MPR } \\
\text { Mean (SD) }\end{array}$ & $\begin{array}{c}\text { Number of individuals } \\
n(\%)\end{array}$ & $\begin{array}{c}\text { MPR } \\
\text { Mean (SD) }\end{array}$ & \\
\hline $\begin{array}{l}\text { All veterans with bipolar disorder on } \\
\text { any antipsychotic with MPR }\end{array}$ & $21,494(38.0)$ & $0.74(0.37)$ & $5,491(31.6)$ & $0.82(0.34)$ & $Z=14.4, p<0.0001$ \\
\hline $\begin{array}{l}\text { Individuals on conventional } \\
\text { antipsychotic }\end{array}$ & $1,700(7.9)$ & $0.81(0.34)$ & $657(12.0)$ & $0.87(0.31)$ & $Z=4.2, p<0.0001$ \\
\hline $\begin{array}{l}\text { Individuals on atypical } \\
\text { antipsychotics }\end{array}$ & $20,514(95.4)$ & $0.74(0.37)$ & $5,044(91.9)$ & $0.81(0.35)$ & $Z=13.6, p<0.0001$ \\
\hline Clozapine & 29 & $0.85(0.24)$ & 9 & $0.81(0.32)$ & $Z=-0.2, p=0.8637$ \\
\hline Risperidone & 6,720 & $0.73(0.36)$ & 1,622 & $0.79(0.35)$ & $Z=6.2, p<0.0001$ \\
\hline Olanzapine & 8,776 & $0.73(0.36)$ & 2,508 & $0.82(0.33)$ & $Z=11.9, p<0.0001$ \\
\hline Quetiapine & 7,410 & $0.76(0.38)$ & 1,400 & $0.83(0.37)$ & $Z=6.2, p<0.0001$ \\
\hline Ziprasidone & 965 & $0.76(0.38)$ & 104 & $0.85(0.34)$ & $Z=2.2, p=0.0293$ \\
\hline Aripiprazole & 114 & $0.79(0.39)$ & 22 & $0.75(0.33)$ & $Z=-0.6, p=0.5349$ \\
\hline $\begin{array}{l}\text { Individuals on a single antipsychotic } \\
\text { medication }\end{array}$ & $17,261(80.3)$ & $0.73(0.37)^{\mathrm{a}}$ & $4,655(84.7)$ & $0.81(0.34)^{\mathrm{b}}$ & $Z=13.6, p<0.0001$ \\
\hline $\begin{array}{l}\text { Individuals on two antipsychotic } \\
\text { medications }\end{array}$ & 4,233 (19.7) & $0.77(0.36)$ & $836(15.2)$ & $0.85(0.34)$ & $Z=5.6, p<0.0001$ \\
\hline \multicolumn{6}{|l|}{ For individuals on only one AP: } \\
\hline Conventional & $\begin{array}{r}10,294(94.4) \\
967(5.6)\end{array}$ & $\begin{array}{l}0.13(0.37) \\
0.80(0.33)\end{array}$ & $\begin{array}{r}4213(90.5) \\
442(9.5)\end{array}$ & $0.86(0.31)$ & $Z=12.8, p=0.0010$ \\
\hline Clozapine & $19(0.12)$ & $0.90(0.15)^{\mathrm{e}}$ & $7(0.17)$ & $0.90(0.14)^{\mathrm{f}}$ & $Z=0.1, p=0.9539$ \\
\hline Other atypicals & $16,275(99.9)$ & $0.73(0.37)$ & $4206(99.8)$ & $0.81(0.35)$ & $Z=12.8, p<0.0001$ \\
\hline
\end{tabular}

${ }^{\mathrm{a}}$ single antipsychotic $v s$ two antipsychotics: Wilcoxon Test, $Z=6.1, p<0.0001$.

${ }^{\mathrm{b}}$ single antipsychotic $v s$ two antipsychotics: Wilcoxon Test, $Z=2.6, p=0.0082$.

${ }^{c}$ atypical vs conventional APs: Wilcoxon Test, $Z=5.7, p<0.0001$.

$\mathrm{d}_{\text {atypical }} v s$ conventional APs: Wilcoxon Test, $Z=2.6, p=0.0094$.

${ }^{\mathrm{e}}$ clozapine $v s$ other atypicals: Wilcoxon Test, $Z=1.9, p=0.0545$.

f clozapine $v s$ other atypicals: Wilcoxon Test, $Z=0.4, p=0.6681$.

assumed an important place in the treatment of individuals with bipolar illness across the age span. Individuals prescribed a single atypical antipsychotic agent in both older and younger groups were less adherent with treatment compared to individuals prescribed a single typical agent. However, the group of individuals on typical agent monotherapy represented a small proportion (approximately $5 \%$ of younger patients, and $10 \%$ of older patients) of all patients. Possibly, individuals on typical antipsychotics represent a sub-group of individuals who have both good response and tolerance to these older agents. Clinicians and patients may be reluctant to switch from therapies that appear to be working well. Interestingly, treatment adherence appears better with two antipsychotic agents compared to monotherapy in both older and younger individuals. This has been observed in other bipolar populations (Sajatovic et al., 2006) in a prospective mixed-age study. It is not clear if more adherent patients are being more aggressively treated, or alternatively, if more aggressive use of atypical antipsychotic agents actually promotes better treatment adherence among patients with bipolar disorder. Clinicians may treat patients with two agents only if they are convinced that patients are adherent with monotherapy, while at the same time avoiding use of complicated (multidrug) regimens for individuals with a history of non-adherence.

Limitations of this study include the fact that prescription refills may not actually reflect medication ingestion, and that the elderly group may represent a survivor cohort characterized by long-term adherence. The 'younger' sample in this analysis averaged 48 years of age. Differences among older $v s$ younger bipolar groups might be more pronounced among bipolar adults in their 20s and 30s vs an older population. Additionally, since this was a VA sample, results cannot necessarily be extrapolated to a more gender heterogenous bipolar population.

It has been suggested that focused attention to treatment adherence may improve adherence (Scott and Tacchi, 2002). Interventions to enhance treatment adherence among older adults with BPD should consider those who are at greatest risk of nonadherence - individuals with comorbid substance use, 
and those with poor psychosocial supports. Additionally, older individuals with BPD are noted to have substantial deficits in knowledge regarding medications and treatment (Schaub et al., 2001) and are likely to benefit from interventions that will improve illness understanding.

\section{ACKNOWLEDGEMENTS}

This work is supported by National Institute of Mental Health P20066054-01A1 and R21MH-62650, National Institute of Mental Health Career Development Award K23MH065599-01A2, and R21S by the Stanley Medical Research Institute.

\section{REFERENCES}

Aagaard J, Vestergaard P, Maarbjerg K. 1988. Adherence to lithium prophylaxis: II. Multivariate analysis of clinical, social, and psychosocial predictors of nonadherence. Pharmacopsychiatry 21: $166-170$.

Aagaard J, Vestergaard P. 1990. Predictors of outcome in prophylactic lithium treatment: a 2 -year prospective study. $J$ Affect Disord 18: 259-266.

Al-Zakwani IS, Barron JJ, Bullano MF, et al. 2003. Analysis of healthcare utilization patterns and adherence in patients receiving typical and atypical antipsychotic medications. Curr Medical Res Opin 19: 619-626.

Berk M, Berk L, Castle D. 2004. A collaborative approach to the treatment alliance in bipolar disorder. Bipolar Disord 6: 504-518.

Colom F, Vieta E. 2002. Treatment adherence in bipolar patients. Clin Approaches Bipolar Disord 1: 49-56.

Gianfrancesco FD, Rajagopalan K, Sajatovic M, Wang RH. 2006. Treatment adherence among patients with bipolar or manic disorder taking atypical and typical antipsychotics. J Clin Psychiatry 67: 222-232.

Gilmer TP, Dolder CR, Lacro JP, et al. 2004. Adherence to treatment with antipsychotic medication and health care costs among Medicaid beneficiaries with schizophrenia. Am J Psychiatry 161: 692-699.

Hertz RP, Unger AN, Lustik MB. 2005. Adherence with pharmocotherapy for type 2 diabetes: a retrospective cohort study of adults with employer-sponsored health insurance. Clin Therapeut 27: 1064-1073.

Keck PE Jr, McElroy SL, Strakowski SM, et al. 1997. Compliance with maintenance treatment in bipolar disorder. Psychopharmacol Bull 33: 87-91.

Lingam R, Scott J. 2002. Treatment non-adherence in affective disorders. Acta Psychiatr Scand 105: 164-172.

Nehra R, Chakrabarti S, Pradhan BK, Khera N. 2006. Comparison of congitive functions between first-and multi-episode bipolar affective disorders. J Affect Disord 93: 185-192.

Patel NC, Crismon ML, Miller AL, Johnsrud MT. 2005. Drug adherence: effects of decreased visit frequency on adherence to clozapine therapy. Pharmacotherapy 25: 12421247.

Perlick DA, Rosenheck RA, Kaczynski R, Kozma L. 2004. Medication non-adherence in bipolar disorder: a patient centered review of research findings. Clin Approaches Bipolar Disord 3: $56-64$.

Robinson LJ, Thompson JM, Gallagher P, et al. 2006. A meta-analysis of congitive deficits in euthymic patients with bipoalr disorder. J Affect Disord 93: 105-115.

Sajatovic M, Bauer MS, Kilbourne AM, et al. 2006. Self-reported medication treatment adherence among veterans with bipolar disorder. Psychiatric Serv 57(1): 56-62.

Sanchez RJ, Crismon ML, Barner JC, et al. 2005. Assessment of adherence measures with different stimulants among children and adolescents. Pharmacotherapy 25: 909-917.

Schaub RT, Berghoefer A, Muller-Oerlinghausen B. 2001. What do patients in a lithium outpatient clinic know about lithium therapy? J Psychiatry Neurosci 26: 319-324.

Scott J, Tacchi MJ. 2002. A pilot study of concordance therapy for individuals with bipolar disorders who are non-adherent with lithium prophylaxis. Bipolar Disord 4: 386-392.

Valenstein M, Copeland LA, Blow FC, et al. 2002. Pharmacy data identify poorly adherent patients with schizophrenia at increased risk for admission. Med Care 40: 630-639.

Valenstein M, Sajatovic M.Adherence to treatment: a lifecourse perspective. In Bipolar Disorders in Later Life, Sajatovic M, Blow F (eds). Johns Hopkins Press: Baltimore, MD.

Young RC, Murphy CF, Heo M, et al. 2006. Cognitive impairment in bipolar disorder in old age: literature review and findigs in manic patients. J Affect Disord 92: 125-131.

Yu YF, Nichol MB, Yu AP, Ahn J. 2005. Persistence and adherence of medications for chronic overactive bladder/urinary incontinence in the California Medicaid Program. Value Health 8: 495-505. 